\title{
Exploring the Efficacy of Anxiety and PTSD Therapeutic Techniques and Protocols in Practice During Ongoing Terrorism: Evidence From a Focus Group Research
}

\author{
Mooli Lahad (Corresponding author) \\ The Community Stress Prevention Center \\ PO Box 767, Kiryat Shmona 11016, Israel \\ Tel: 972-4-690-0600 E-mail: 1ahadm@netvision.net.il

\section{Dmitry Leykin} \\ The Community Stress Prevention Center \\ PO Box 767, Kiryat Shmona 11016, Israel. \\ Tel: 972-4-6900600 E-mail: $\underline{\operatorname{dimleyk@gmail.com~}}$
}

Ornit Rozenblat

Sha'ar HaNegev Psychological Services Center

D. N. Hof Ashkelon 79200, Israel

E-mail: rozenblat2@,bezeqint.net

\section{Zvi Fajerman}

Sha'ar HaNegev Psychological Services Center

D. N. Hof Ashkelon 79200, Israel

Tel: 972-8-680-2752Ｅ-mail: zvif@sapir.ac.il

Received: March 15, 2014 Accepted: April 23, $2014 \quad$ Published: June 5, 2014

doi:10.5296/ijsw.vli1.5289 URL: http://dx.doi.org/10.5296/ijsw.vli1.5289 


\begin{abstract}
The aim of the present study is to describe the accumulated knowledge and experience of experts working, treating and living under ongoing threat setting, an understudied research topic. Using focus group approach, psychologists, clinical social workers and art therapists, staff members of the Sha'ar HaNegev Psychological Services Center (PSC) located in South Israel, were asked to describe their clinical experience of working with trauma affected children, adolescents and adults. Effective use of techniques and protocols along phases of treatment, indication of less effective techniques, newly developed techniques and clients' experience are reported and discussed.
\end{abstract}

Keywords: PTSD, Anxiety, Ongoing stress, Focus group

\title{
1. Introduction
}

The present study describes the accumulated knowledge and experience of experts working, treating and living under ongoing threat settings. The knowledge regarding effective practices in such setting is relatively scarce (Diamond, Lipsitz, Fajerman \& Rozenblat, 2010) and not comprehensive. Although there is immense progress in the study of psychological treatments for stress related disorders, significant gaps in practical knowledge exist and hinder better understanding for practitioners working in geographical areas prone to constant threat and ongoing terrorism.

For the clinical practitioner, the most fundamental issue in psychotherapy research is that of effectiveness. For any new therapeutic approach, one must ask "Does it work?" (Mahoney, 1977). The complexity of the answer is chained to variation of circumstance, clients, therapists, etc.

The overall aim of the study is to explore the current use of common techniques and protocols in the treatment of stress related conditions, under shared ongoing trauma. The study tries to understand whether in the absence of research on such conditions and their impact on treatment outcome, is it possible to learn from the clinicians? And if it is possible, how often, if at all, do they use Evidence Based Treatment (EBT) protocols. In addition, the study aimed to find out whether practitioners find some tools to be more effective than others, and are there any age specific recommendations. Finally, we asked whether practitioners developed face-value effective methods. Exploring such questions and drawing practical conclusions will help psycho-trauma therapists address the psychological needs exhibited by clients in threat posed areas.

\section{Literature Review}

The present literature review was conducted with the aim to present existing contemporary psychotherapeutic techniques and protocols for Posttraumatic Stress Disorder (PTSD), describe their traditional implementation in clinical practice, and present the need to explore clinical practice in areas prone to constant security threat. We searched databases such as PsycInfo, Sciencedirect, EBSCO and Web of Science with the terms "PTSD treatment" AND "protocols" OR "techniques" AND "ongoing terrorism". Grey literature was not reviewed. 
PTSD is the most known mental disorder associated with experiencing an overwhelming life-threatening incident (APA, 2000), develops only among small subset of trauma victims $(<10 \%)$ (Breslau, 2009). The attention that professional literature dedicates to therapeutic techniques and treatment protocols of PTSD and anxiety disorders is prominent. Foa, Keane, Friedman \& Cohen (2009) present in their practice guidelines dozens of PTSD effective treatments for children, adolescents and adults. These include Cognitive-Behavioral Therapy (CBT) (e.g. exposure therapy, stress inoculation training) Psychopharmacotherapy, Eye Movement Desensitization and Reprocessing (EMDR), Group Therapy, School-Based Treatment, Psychodynamic therapy, Psychosocial Rehabilitation, Hypnosis, Couple and Family Therapy, Creative Techniques (imaginal exposure, cognitive/narrative restructuring) and Creative Arts Therapies (e.g. art, dance, drama, music, psychodrama, and poetry). Cukor, Spitalnick, Difede, Rizzo \& Rothbaum (2009) also review emerging psychotherapeutic and pharmacologic interventions for the treatment of PTSD such as social and family based (e.g. interpersonal psychotherapy), behavioral (i.e. behavioral activation, trauma management therapy, interoceptive exposure, mindfulness, yoga and acupuncture), imagery-based (i.e. imagery rescripting, imagery rehearsal), therapies focusing on distress tolerance (i.e. DBT, acceptance and commitment therapy), "power" therapies (i.e. thought field therapy, trauma incident reduction, vision kinesthetic disassociation) and technological-based treatment (i.e. internet and computer based treatments, virtual reality). Another emerging effective integrative treatment protocol which combines CBT, imagery-based and body-mind methods is SEE FAR CBT (Lahad, Farhi, Leykin \& Kaplansky, 2010). However almost all the methods have been designed and tested post events as indicated by the term Post traumatic. Even those who treated long term exposure such as the Holocaust treated their patients' years after the 'real' horrifying event has ended.

According to the International Society for Traumatic Stress Studies (ISTSS) practice guidelines, each treatment might fall into one of six categories of endorsement in a coding system, which represent varying levels of evidence for the use of that specific mean of intervention and varying from evidence that has not been subjected to clinical or empirical tests in PTSD (criterion F), and to evidence based on randomized, well-controlled clinical trials for individuals with PTSD (criterion A) (Foa et al., 2009). Past literature shows that the most effective treatments cited are prolonged exposure (PE), and eye movement desensitization and reprocessing (EMDR) (Seidler \& Wagner, 2006). More recent findings show continued support for the efficacy of individual trauma focused CBT (TFCBT) and EMDR for the treatment of chronic PTSD (Bisson, Roberts, Andrew, Cooper \& Lewis, 2013). Those treatments have proven successful in changing and bettering the situation of trauma victims (Mendes et al., 2008). Although dozens of studies and trials have proven the efficacy of some leading evidence-based treatments (EBT) for PTSD earlier mentioned and majority of trauma specialists support EBT practice generally (Gray, Elhai \& Shmidt, 2007), manulized interventions or evidence based treatments are poorly implemented in clinical practice (Becker, Zavfert \& Anderson, 2004; Cahill, Foa, Hembree, Marshall \& Nacash, 2006; Waller, 2009; van Minnen \& Hendriks, 2010). Among the common reasons mentioned in the above works to serve barriers for implementations of EBTs and EBPs for PTSD in public-sector practice settings were lack of training in the techniques, hesitancy and 


\section{Macrothink}

International Journal of Social Work

ISSN 2332-7278

2014, Vol. 1, No. 1

disinclination to use protocolized treatment and concerns about decompensation. Frueh, Grubaugh, Cusack \& Elhai (2009) noted that implementation of evidence based practices is critical to improving patient outcomes, and Cukor, Olden, Lee \& Difede (2010) concluded that "failure of providers to use established treatments is a barrier to effective care that as a community must be addressed" (p. 87).

Though considerable research on PTSD symptomatology and treatment following terrorist attacks or war is based on studies post incidence or postwar (Cloitre, 2009), there are reports on the mental health problems of civilians living under conditions of ongoing threat (Al-Krenawi, Graham, Kanat-Maymon, 2009; Besser \& Neria, 2009; Farchi \& Gidron, 2010; Freedman \& Tuval-Mashiach, 2006; Diamond, Lipsitz, Fajerman \& Rozenblat, 2010; Thabet, Tawahina, Sarraj \& Vostanis, 2008; Thabet, Ibraheem, Shivram, Winter \& Vostanis, 2009; Lahad \& Leykin, 2010).

Over the past 14 years, the Israeli southern town of Sderot and its neighboring surroundings have been prone to ongoing shelling and missiles attacks from the proximate Gaza strip. In this current reality, there are almost no intermissions, and ceasefires are usually brutally disrupted. The sequel is over 12,800 rockets (Grads, Qassams) and mortars launched at Israel, an average of 3 attacks every single day, leaving hundreds emotionally and physically wounded (IDF Blog, 2014). The deployment of the "Tzeva Adom" ("Red Code") early warning system in the cities of Sderot, Ashkelon and other at-risk targets paved a tense daily routine for civilians, who in the moment of truth have between 15-45 seconds to take cover before impact (for in detail description see Diamond, Lipsitz, Fajerman \& Rozenblat, 2010). Some of the civilians have managed to assimilate this peculiar condition in their routine, as suggested by previous study exploring routine maintenance during ongoing exposure to terrorism (Pat-Horenczyk, Schiff \& Doppelt, 2006), while others have been struggling and eventually developed intense prolonged psychological reactions. As a result, depression and anxiety diagnoses rose between the years 2001-2006, leading also to higher consumption of antidepressant drugs (Farhi, Lauden, Ifergan \& Fariger, 2008). With that, recent studies report $27 \%$ of a probable clinical Post Traumatic Stress Disorder (PTSD) among adult population in this area (Besser \& Neria, 2009), and 62.9\% of PTSD among children (Farhi \& Gidron, 2011) in a non-clinical population. Another study shows that approximately $79 \%$ of the representative clinical sample $(\mathrm{N}=117)$ of Shaar Hanegev Psychological Services, located in the surroundings of Sderot, have an above moderate-severe level, as measured by accepted self-report PTSD diagnostic scales (Lahad \& Leykin, 2010).

In order to describe the clinical picture of PTSD in ongoing threat settings, Diamond et al. (2010) suggested the term Ongoing Trauma Stress Response (OTSR), which describes a distinct clinical representation than PTSD. First, patients report of gradually increasing anxiety symptoms, and anticipation of future attacks, threat of injury or death. Second, they are less likely to present re-experiencing and intrusion symptoms, and instead are preoccupied with fears associated with their daily activities and with hyper-arousal symptoms. Third, client's fears and patterns of avoidance are typically reality based or at least reasonable - thus showing normal patterns of behavior in abnormal situation (Shalev, Tuval, Frenkiel-Fishman, Hader, \& Eth, 2006). Fourth, they show amelioration in most reported 
symptoms (aside from startle response) when there is lull in violence or when they are out of the rockets range. Similarly to PTSD, OTSR can significantly impair everyday functioning and quality of life. Diamond et al. (2010) described set of functional impairments derived from the proposed clinical picture, for instance: chronic insomnia, absenteeism, problem concentrating at work, decrease in exercise, reduced social support, family problems and more.

As mentioned before, there is abundant research on the treatment of PTSD in clinical setting post incident (Foa et al., 2009), but there is not much evidence in the literature for PTSD psychotherapy practice under ongoing and prolonged threat of terrorism or war. A recent published study (Diamond et al., 2010) describes a single case presentation of an adult woman leaving in Sderot, suffering from OTSR. The researchers described her course of therapy, which included cognitive reframing, providing psycho-education, teaching mindfulness skills and practicing in-vivo exposure, eventually lessening her baseline anxiety. Although it was previously proposed that trauma-focused psychotherapy may not be advisable to patients who continue to be exposed to traumatic events, and establishment of safety and security in the initial state is proposed (Foa et al., 2009), recent reports show that individuals exposed to ongoing and intense traumatic war-related stress can benefit from intervention. For instance, preliminary unpublished data from Sderot shows that PTSD patients managed to reduce their posttraumatic symptoms following SEE FAR CBT treatment, even though exposed to reoccurring terrorist attacks (Lahad, Doron, Farchi, \& Leykin, submitted). Berger, Gelkopf \& Heineberg (2012) found that adolescents from Sderot who received 16 sessions of a teacher delivered intervention, significantly reduced posttraumatic stress-related symptoms, somatic complaints, functional impairment, and anxiety.

Even though we tend to agree with Foa et al. (2009) proposition that trauma-focused psychotherapy may not be advisable to patients who continue to be exposed to traumatic events, still the ethics of therapists dictate intervention and as the literature does not indicate what to do, in practice all the known EBT methods (and others) are being used with patients who continue to be exposed to traumatic events. In the case of Sderot and the surrounding area, the situation is even more complex as the therapist and the client maybe under shelling whilst the treatment takes place, or the therapist and the client might have been exposed to the same threats at home. Both therapist and client are in a relative safe place (the shelter of the trauma center) but their loved ones are somewhere in the area exposed to the threat. Thus in the current shared reality of ongoing threat (Baum, 2013), an urgent need raises to examine what practice is helpful, what out of all the 'toolbox' of psychotrauma EBT methods is working, and are there new methods to overcome barriers in treatment caused by recurrent attacks. For instance, our clinical experience suggests, that the deployment of imaginative techniques in establishing a safe place during treatment was found effective (Lahad et al., 2010). It is important to ask what is the accumulated knowledge and experience of experts working, treating and living in this complicated situation.

For the present study, our main hypothesis is that clinicians working under constant threat would introduce new therapeutic methods and techniques they found useful and effective. We further propose that clinicians would show greater use of treatment techniques rather than 
treatment protocols.

\section{Methods}

\subsection{Participants}

Twenty seven psychologists, clinical social workers and art therapists, staff members of the Sha'ar HaNegev Psychological Services Center (PSC), located in Sderot, Israel, participated in three meetings: August 2008, August 2009, and follow up meeting in 2012. Holding in mind that the PSC on a daily basis consists of approximately 30 clinicians, the present sample is appropriate to represents the views of clinicians working in Sderot. Those participants (79\% women) had a median age of 46.5 (range 34-82), median of 15 years in practice (range 3-45) and median of 11 years (range 1.5-45 years) in the psychological services center. Participation was part of an in-service training given out by the PSC.

\subsection{Data Collection Method}

In the current investigation we used the focus group approach to collect the data. Kitzinger (1995) notes that this process is particularly valuable for exploring and investigating people's knowledge and experiences and can be used "to examine not only what people think but how they think and why they think that way" (p. 299). According to Kress \& Shoffner (2007) focus groups qualitative research methodology has been a suitable and "effective means of understanding the counseling process and clients' needs and experiences in counseling" ( $\mathrm{p}$. 189). A survey of 133 outcome studies, published between 1996 and 2000 in a leading research journal, found that only $23 \%$ utilized therapist-derived data (Hill \& Lambert, 2004). Daniel and McLeod's (2006) findings suggest that practitioners may possess sensitivity to the complexity of outcome that is missing in much current research. Consequently to the absence of research which has made use of the practitioner's perspective on outcome in the field, Daniel and McLeod's (2006) study explored the implicit and informal construction of outcome evaluation by experienced practitioners via interviewees in which they talked about four different types of information that they regard as relevant to their understanding of success: Client satisfaction, evidence of change, the counseling relationship and personal satisfaction.

An introduction session was carried out by one of the authors (ML), explaining the rational for engaging the process. Next, clinicians filled out a background structured survey, which aimed to obtain initial information about the frequency of using the therapeutic protocols and methods. They were presented with seven different contemporary interventions (PE, SE, EMDR, SEE FAR CBT, Family Therapy, Dyadic Therapy and Dynamic Therapy) and were request to rate the frequency of their usage in the protocol on a 3 point scale (ranging from 1-never, 2-as required, 3-every session), their impression regarding protocol's efficacy on a 5 point scale (1-not effective, 2- low 3-moderate, 4-effective, 5-highly effective) and the age groups which they applied the protocol to (infancy to 5 years, 6-11 years, $12-18$ years and 18 years and above). Later, twenty-two therapeutic techniques were presented (e.g. guided imagery, relaxation, breathing techniques, in vivo exposure) with three focus questions: 1) frequency of usage, rated this time on a 5-point scale (1- never, 2-rarely, 3- sometimes, 


\section{Macrothink}

4-often, 5-almost every session); 2) perceived effectiveness of the techniques (same rating procedure as for the therapeutic protocols); and 3) target clinical population. For each section an "other" item was offered in order to cover additional clinical and therapeutic procedures being incorporated in practice.

Next, they were divided into five small discussion groups which included between 4-7 members, according to of the age of their 'target patients' (e.g. one group discussed issues regarding patients under 5 years old, another group discussed issues regarding patients 6-11 years old). For each small focus group participants were asked to describe: 1) common emotional, physiological, cognitive and behavioral symptoms of the patients; 2) possible subdivision of the patients according to their clinical picture; 3) perceived effective therapeutic techniques in the beginning, middle and the end of the therapeutic process; 4) new methods and techniques which were brought up during the clinical practice; 5) helpful experiences or methods which were reported by patients during therapy; 6) less effective techniques in practice; 7) helpful tools for the therapeutic practice; and 8) potential factors for prediction of therapy success. In each small discussion group one clinician was instructed to take notes and fill out an open ended survey with the answers and ideas given by other members of the group.

\section{Results}

\subsection{Use of Evidence Based Treatment Protocols}

All therapists reported that they used at least one of the various common therapies However as Figure 1 shows the most frequent method in use is Dynamic therapy (90\% reported they used it as required or every session). SE (82.6\%) and SEE FAR CBT (80\%), that are non EBT but still use protocol and quasi experimental methods to support their efficacy were among the frequent protocols in use. Of the EBT protocols EMDR (31.6\%) and PE (47.6\%) were used the least. However, when counting the number of responders assessing the efficacy of these protocols, it is revealed that probably only very few therapists were trained on the methods that were the least in use. For instance, 18.5\% and 37\% assessed EMDR and PE for their efficacy, while 70\% assessed SE and Dynamic therapy for efficacy. 


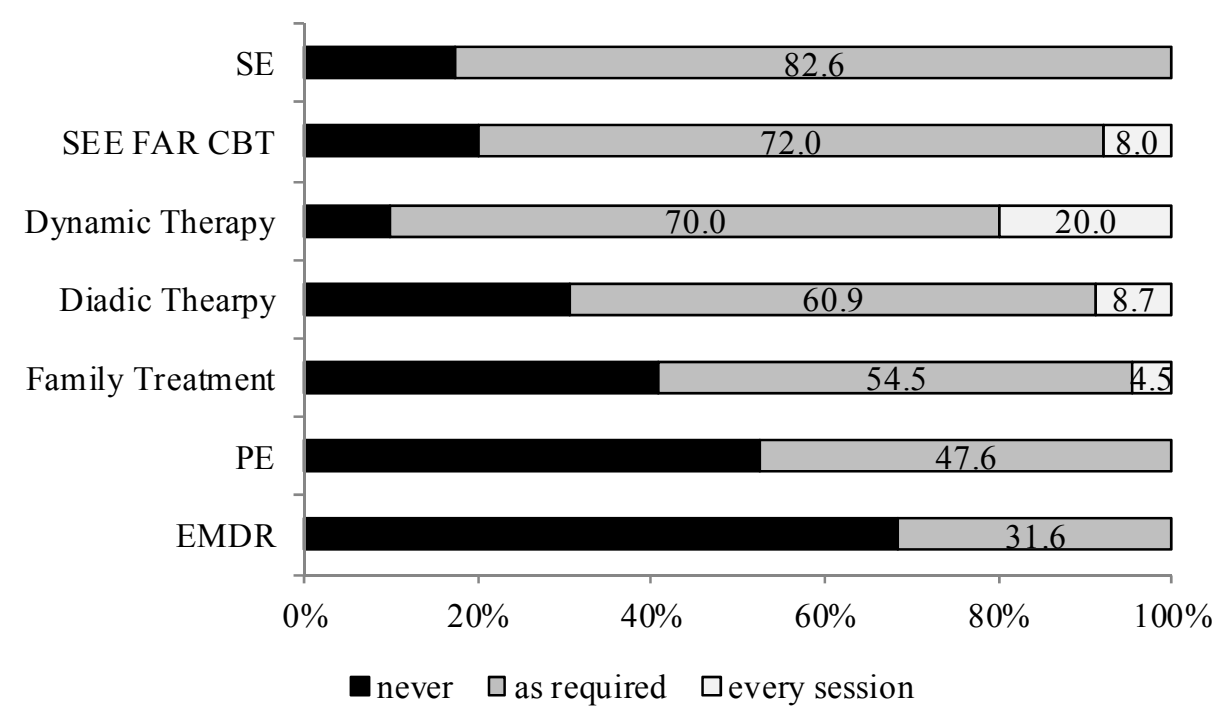

Figure 1. Use of various methods and protocols

\subsection{Effective Use of Techniques and Protocols Along Phases of Treatment}

Figure 2 summarizes frequency of techniques usage during treatment sessions as indicated by the therapists, as function of clients' age group. 


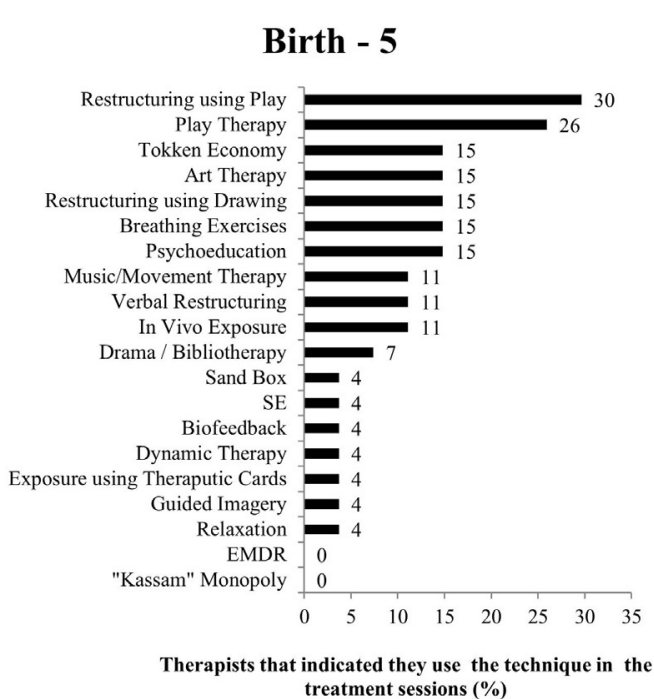

$12-17$

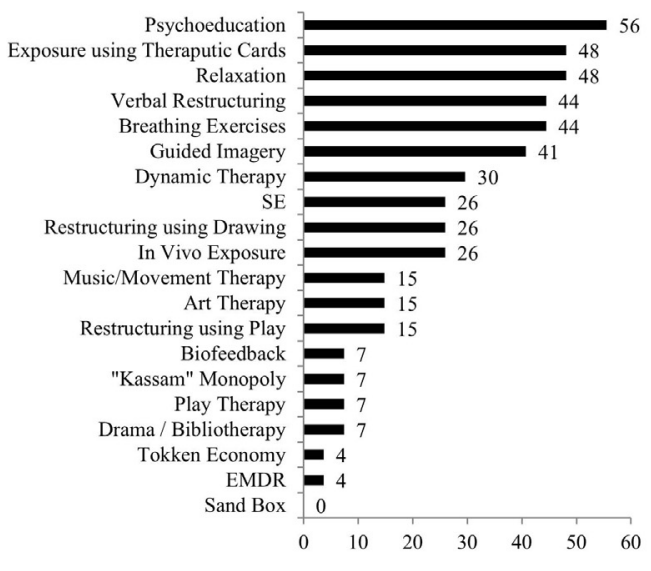

Therapists that indicated they use the technique in the treatment sessions (\%)

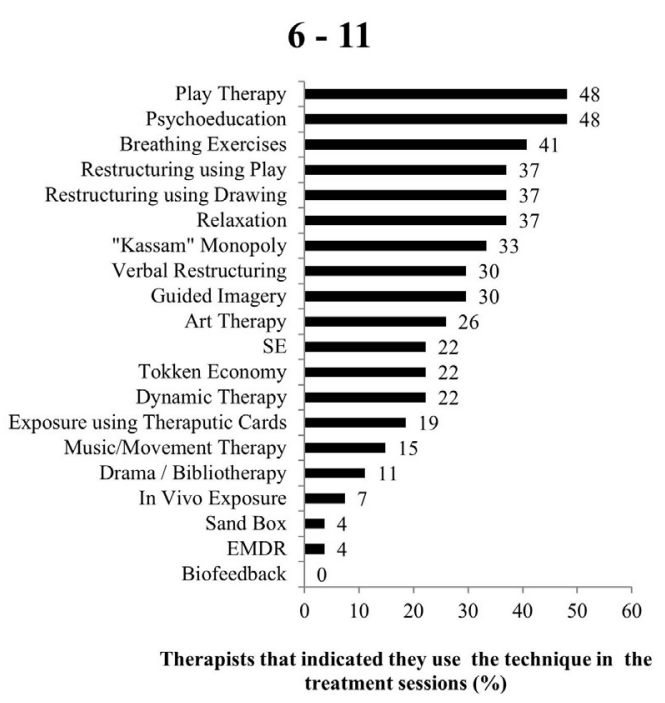

$18<$

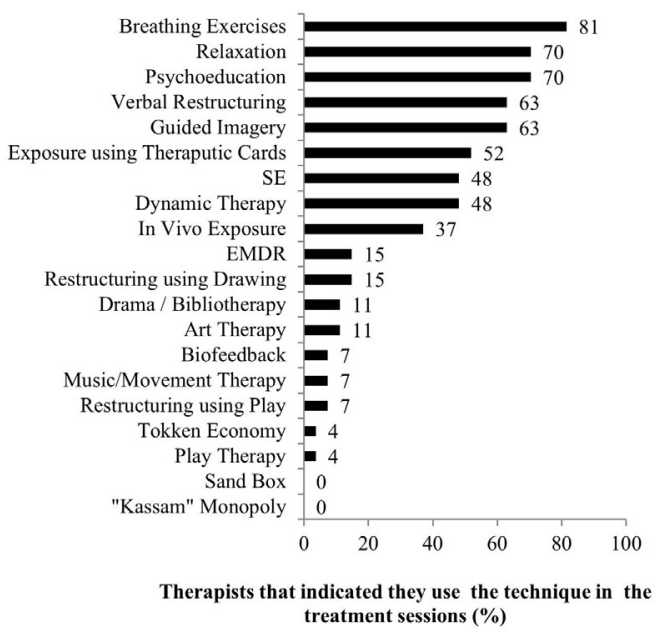

Figure 2. Frequency of technique usage in the treatment

For young children it was reported that experiencing "Red code" together with the therapist and the experience of the treatment in a safe place were helpful for the patients as it probably made the in-vivo experience an opportunity for the child to gain support for the "prescribed" behavior (deep breath, jumping, singing) and for the therapist to be able to respond on the spot to the child's - parents behavior, but more so the therapists believe that this was a bonding experience and an opportunity for modeling for the parents how to handle the situation including admitting that this was fearful experience without crumple. In addition, constructing normalized schemes (i.e. "I am well and my child is well"), and permitting parents and children a place to discuss and process together what is happening during and after emergencies was reported as an empowering effect. 


\section{Macrothink}

Therapists reported that for children (6-11) the daily practice of breathing exercises, psycho-education, token economy, guided imagery, in vivo exposure, concrete practice of behavior during 'code red' and parental psycho-education was very helpful.

Adolescents report that the certainty about therapist existence, therapist's expressions of care after security events (including a phone call and text messages), psycho-education mostly sharing the rational for every intervention, breathing exercises and guided imagery were especially helpful during the various phases of treatment.

As for Adults $(18<)$, it was practicing relaxation, breathing, use of therapeutic cards as a mean for creating an imaginal safe place, normalization, psych-education, pendulation (in EMDR and SEE FAR CBT) and available communication channel with the therapist (e.g. phone call or texting) during emergency events and escalation.

\subsection{Less Effective Techniques}

Therapists reported that trauma reconstruction using therapeutic cards in children up to seven years is not effective, since they tend not to understand the procedure. In addition, mutual (parent and child) reconstruction of the traumatic story was less effective when the parent is still overwhelmed by the events. It was also reported that when one or both parents suffer from depression, or when other siblings suffer from a significantly more serious problems this creates a significant barrier for dyadic therapy. As for children aged 6-11 it was noted that SE is less effective. Among adolescents, it was reported that being over inquisitive was perceived by the patient as bothering, and excessive behavior reinforcement had similar effect. Among adult patients it was reported that stiff adhering to a single technique along all treatment stages hindered treatment outcomes.

\subsection{Perceived Effectiveness of Treatments and Techniques}

Figure 3 describes the perceived efficacy of techniques, categorized into three groups: not effective or low effectiveness, moderate effectiveness, effective or highly effective. Only techniques that were rated by more than 10 therapists are presented. Techniques such as Reflexology, Sandbox, Biofeedback, Drama / Bibliotherapy, Music, EMDR techniques and Art Therapy techniques were excluded, though qualitative description regarding their efficacy was collected from some therapists. 


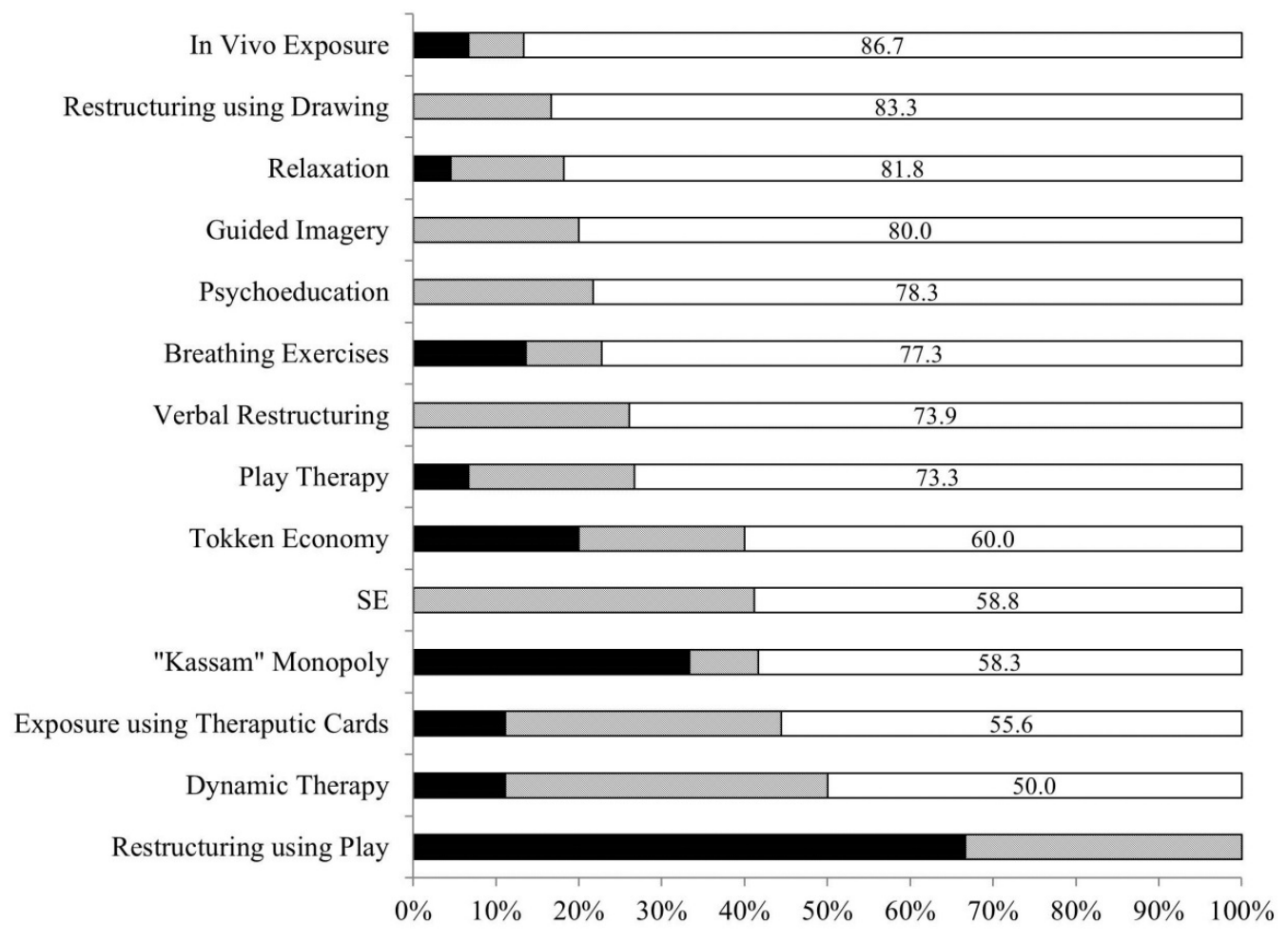

not effective or low $\square$ moderate $\quad \square$ effective or highly effective

Figure 3. Perceived effectiveness of treatment techniques

In overall, at least $75 \%$ the therapists reported that in vivo exposure, restructuring using drawing, relaxation, guided imagery, psycho-education and breathing exercises were effective or highly effective. For other treatment techniques, such as Dynamic Therapy techniques, exposure using therapeutic cards and more newly developed techniques (e.g. Kassam Monopoly) received less consensus regarding their effectiveness. Restructuring using play technique was mainly rated as not effective or low effectiveness.

\subsection{Newly Developed or Additional Techniques Being Implemented During Therapy}

\subsubsection{Techniques for the Youngest Group}

a) "Koko Taki" a card game that confront the players with various stressful as well as helpful situations thus enabling the clients to 'touch' upon unspoken situations and manage it through the game.

b) Singing together in the protected space (Merkhav Mugan)

c) Kassam Monopoly, a table game based on the original monopoly but deals with the situation and using the rocket's name Kassam both as the games name and as a desensitizing element as it is used in a humoristic game. 


\section{Macrothink}

d) "Security Box" box that was created together with client with all the things that can help in times of stress (practical, recreational, and imaginative (adapted from Ayalon \& Lahad, 2000)

e) Structuring mutual story of Parent \& Child

f) Multidisciplinary work along with paramedical staff and kindergarten staff

4.5.2 Techniques for Children Aged 6-11 Years Old

a) Drama, drawing and storytelling techniques

4.5.3 Techniques for Adolescents (12-17)

a) Using virtual communication (e.g. email, text messaging) as an alternative to disruption of setting.

b) "Courting"- calling the patients when they don't come, writing letters, active follow up and outreach.

c) Using therapeutic cards (Lahad et al., 2009) as sublimative means.

d) Treatment at alternative place ( that is keeping the appointment but not necessarily in the usual setting)

4.5.4 Techniques for Adults

a) Pairing "Red Code" with coping activities (e.g. breathing and relaxation)

b) Modeling of clam body language

c) Narrative methods of story restructuring and reframing together with the therapist

d) Empowerment of patient's adaptive self-behavior and capacity

e) Emphasizing treatment progress, on each step.

4.6 Effective Activities Reported by the Clients During Times of Recurrent Threat When out of the Clinic

Besides the techniques mentioned earlier, participants report that for the young children (birth -5 years) breathing exercises during moderate subjective levels of distress (not reaching high levels), reducing of muscles tension were helpful and effective.

For the children group it was reported that breathing exercises, physical exercises, guided imagery were helpful. Additional resources included community, family and social support, keeping on normal daily routine, normalization of anxiety, presence of an animal and acquaintance with existing safe place (e.g. bomb shelter or protected space).

Participants, who mainly worked with adolescents, reported that mostly physical activity and social activity (e.g. shopping) were helpful during times of recurrent threat when out of the clinic. 
Adults mentioned the community, social and family support to be significant assistance. Having faith in the leadership, receiving information, contact with relatives outside the area, going on respites, and close friend that I can talk to as well as breathing and relaxation were helpful out of clinic activities as well.

\section{Discussion}

The purpose of this study was to fill a gap in the field of Psychotrauma dealing with the treatment of PTSD symptoms of clients exposed to ongoing traumatic incidents.

Indeed the field of Psychotrauma treatment gained a lot of credit since the introduction of evidence based research leading to evidence based treatments (EBT). With national guidelines such as the National Institute for Clinical Excellence (NICE) (2005) guidelines suggesting that "The guideline recommendations have been developed by a multidisciplinary team of healthcare professionals, PTSD sufferers and guideline methodologists after careful consideration of the best available evidence" (p. 9).

To-date there are various guidelines such as VA/DOD Clinical Practice Guideline For The Management of Post-Traumatic Stress (2004), and the International Society for Traumatic Stress Studies guidelines (Foa et al., 2009), all are based on methods that were proven effective in reducing symptoms. In fact, Foa et al. (2009) suggest that trauma-focused psychotherapy may not be advisable to patients who continue to be exposed to traumatic events, and establishment of safety and security in the initial state is proposed. As mentioned above these methods are all post event, individually focused in most cases, and in that respect do not have the practice nor the research to provide guidelines for the treatment of long term or ongoing exposure to traumatic incidents nor do they cover incidents where both clients and therapists share the same experience and threats to themselves and their loved ones for years.

The unusual conditions in some low intensity conflict areas (such as the ongoing conflict in the south of Israel and the Gaza strip) where traumatic incidents are an ongoing situation (for almost 14 years), lead to high prevalence of PTSD symptomatology (Al-Krenawi, Graham, Kanat-Maymon, 2009; Besser \& Neria, 2009; Farhi \& Gidron, 2011).

The attempts to train and support the introduction and the application of EBT were based on the international call for the use of such methods in the treatment of PTSD. However the absence of EBT methods that deals with the above circumstances challenge the duty of community mental health to support, help and alleviate suffering for clients. With this in mind we carried out this study.

We learned that therapists are happy to learn new methods and protocols probably as a mean to help themselves to counter their own helplessness and as a mean to be part of the international trend but are reluctant to use full protocols or are frustrated once the protocol lead to expected outcomes but with the first relapse the therapists and the clients lost confidence in this approach. As one practitioner said "how do you send a client to practice in vivo of going out to throw the garbage when you know the probability of recurrent attack is high?" or another stated "how do you encourage a client to go to a mall where he used to shop when you (therapist) know there is no shelter?". Therapists' complaint that most of the 
protocols did not include instructions for such relapses and found that to be rather frustrating.

Foa, Gillihan and Bryant (2013) in their attempt to study why EBT method aren't used by practitioners, suggested that in most cases it is due to lack of confidence in structured/directive approach mostly based on the therapists' previous psychodynamic training: professional culture that often is antagonistic to evidence-based treatments (EBTs), mental health professionals that prefer individualized to manualized treatments, mental health professionals who do not follow expert guidelines. We found that these explanations were evident in our discussion groups when the therapists were very experienced and dynamically oriented. Foa et al. (2013) also mention effective dissemination models, but in the case at hand this was not the issue as there was ample supervision and the management of the center supported the incorporation of the EBT methods. Some other explanations are therapists' being frightened to re-expose clients to their suffering or finding it too harsh to re-expose them (using the imaginal exposure element) as one therapist said: "how could I put her through this frightening event again it will harm her ability to function and to sleep at night".

So it seems that the therapists opted in most cases to use elements of the protocols that were easy to adapt and integrate into their existing methods of psychotherapy. Those who used full protocols and have seen their positive effect tended to use them more, however they also reported frustration when clients returned to treatment after a successful reduction in symptoms due to recurrent exposure to traumatic incident, as they feared they can't re-use those methods again. It is noteworthy that the methods that were reported to be effective are common to most EBT protocols. These methods were: psycho education, breathing, relaxation, verbal retelling, in vivo, modeling, image-cards exposure and re-narration of the incident.

Indeed this was a clear shift from the traditional psychodynamic nondirective approach and therapists explain the incorporation of these methods for three reasons: their own need to alleviate clients' suffering, the client's demand for something practical and the fact that the above methods could be relatively easy to adapt to their existing style of therapy. Cohen et al. (2013) stated that evidence based therapy models developed to treat traumatized children, such as Trauma Focused CBT (Cohen et al., 2000), are limited in their focus to helping children process a single traumatic event. They suggest that the ongoing exposure to traumatic situation calls for a special methods of working with early childhood, incorporating dyadic methods to help both parent and child. This same approach was evident in the current study where therapists noted that early childhood needs to include a parent-child approach and the need for developmentally appropriate methods, such as play and games. The fact that the therapists invented new games (e.g. Kassam monopoly) show that structured work is slowly becoming part of the therapeutic methods. We would recommend that a protocol such as the above mentioned TF-CBT method (Cohen et al., 2000), be adapted to the ongoing situation. This is mostly as this method has been widely researched with various populations and as an EBT method, it is replicable and its' content uses variety of modalities that were mentioned by the early childhood and the 6-11 age group in our study, though in the current situation are not part of a protocol. 
It is clear that with the older population therapists were more willing to use full protocols (CBT, SEE FAR CBT) or parts of the structured protocols and saw more positive results. However, it is fair to say, though on a lower degree, that even in this group, therapists found it difficult to "re use this protocol when client comes back with relapse symptoms sometimes even worse, as they feel that even these effective methods can not cure them".

In fact in the 2012 follow up interviews carries by the ITC (unpublished) clinicians opted for what they called "therapeutic tool box" corresponding with (Diamond et al., 2010), who suggested that those who struggle to cope under constant sense of threat, and show distinct phenomenology of psychological suffering, may benefit from the eclectic application of therapeutic techniques rather than focusing on a single treatment protocol.

We recommend expert group on the treatment of PTSD to invest in the study and development of EBT protocols for treatment of psychotrauma in ongoing exposure to multiple sources of familial and community violence, or to prolonged situations of war and recurrent terrorism. Given that there is limited evidence based treatment options for those with Ongoing Trauma Stress Response, we recommend among other issues that the protocol/s should look into a wide range of functional problems that set various needs for the affected individuals. For instance, they need more guidance on maintenance of good health and integrating effective methods of self-relaxation (e.g. breathing exercises and guided imagery) to improve quality of sleep and concentration problems. Clients need to be motivated to initiate activities and be instructed on effective coping techniques that they can implement outside their home to gain sense of control, reduce absenteeism and alleviate participation in different activities. Involvement of significant others in the treatment can assist in treatment compliance, promote reconnection to social networks and find acceptable solution to potential family problems that were created due to the psychological condition of the patient. Finally, the inclusion of the education system in the treatment warrants further attention as well. To summarize this part we suggest that first line treatment should be an EBT protocol with flexibility to add creative modalities and optional other methods to support its efficacy.

Another aspect of this study dealt with the therapists themselves living in the same area and worried for the safety of their loved ones. When asked to mention what helped them personally to work in this extreme situation, therapists mentioned peer support, inter disciplinary work with the medical team, group supervision, group introspection and sharing personal experience. Additional sources of support included professional maintenance, learning more techniques and getting more experience in using them, including treatment focused supervision, getting information as to which treatment fits for which situation.

Recent research suggests that the constant uncertainty and threat create emergency routine for the clinicians and can affect them in several domains (Baum, 2013). As the clinicians have significant roles that oblige them to stay in the threat zone and continue to provide psychological treatments. Emotional exhaustion and work-family conflicts reported as intensified and affect the professional competency of the clinicians. We suggest, that such pressures may lead to reduced motivation to monitor the effectiveness of techniques and reluctance to integrate protocols and techniques that were proved to be effective, as they 
demand adherence and reports that at least in the initial stages are time consuming and thus clinicians opt for 'treatment as usual' rather than new and effective protocols. Still we wish to emphasize that none of the existing EBT methods addressed the phenomena of ongoing incidents more over none of them relate to incidents where the therapists and their own families are exposed to the same ongoing threats as their clients.

Albert Einstein once quoted Plato that said: Necessity is the mother of all invention. Indeed in the absence of specific methods to treat children the therapists used their own creativity and invented new methods. It seems that the ones that appeared to be helpful for the early childhood group, included game type activities such as concrete problems solving cards games, a "safety box" for parents ready to be taken with them to the safe room in order to keep child busy, joint story-making with child and parents. For the latency age, the use of drama, story-making methods, improvisation, drawings and the box game "Kassam monopoly". For the early puberty group using the internet as alternative communication, and the use of therapeutic cards. It looks as the clinicians transformed known methods of playtherapy in which they felt confident adapting known games to deal with emotionally loaded issues. As one of them said "Using table game with set of rules, it was easy to visit uneasy topics. The notion that we were "only playing" thus whatever comes out of the cards or by the dice's is a matter of chance made it less threatening or intrusive. Yet as part of the "game's rules" the issues were raised and the players had to 'react' to them".

When asked what are the indications for therapy termination the responses confirmed indicators that are used by EBT methods (Foa et al., 2009). This may indicate that some aspects of the training in EBT protocols were assimilated. They reported the following indicators: returning to normative functioning, reduction in symptoms, better communication with adults and especially with parents, parental ability to contain their child's difficulties and self-report on in vivo management during "red code". All therapists suggested follow up by themselves on clients' adjustment and function.

All clinicians referred to the special situation they called Ongoing Trauma Stress Response (OTSR) and acknowledged the fact that participating in this inquiry process was very helpful and validating experience that gave them a sense of importance and empowerment.

In a follow up survey held by the Israeli Trauma Coalition (a consortium of professional psychotrauma treatment NGOs) (2012) the reports on effective treatments in the same area showed the same results. However this time, four more years of being under on-going stress of shelling past, adding to the therapists and clients mounting distress. The therapists were mostly concern with relapse. They reported 30\% relapse that challenges their skills and lead to think differently on the way they treat patients in this area. Their conclusion opted for a "tool bag" rather than a protocol. The few that reported using EBT protocols (SEE FAR CBT, EMDR and SE) were asking for a manual for long term or ongoing situations of stress and trauma, as they found no literature on how they can use the EBT protocols over and over again with those who demonstrate relapse. 


\section{Limitations and Future Directions}

Though therapists' qualitative reports may reveal important professional knowledge that should be translated into practical implications, the study also have several limitations. First of all, self-report data was earlier reported to have some validity problems (Barker, Pistrang, Elliott, 2003), and it was recommended to interpret such information in caution. In addition, it was suggested that in order to validate self-report data, it should be integrated with observational data (Barker et al., 2003). Although, some research had already shed light on the efficacy of certain PTSD treatment protocols, more cross-validation studies are needed to confirm the findings found in the present work. Second, a potential bias in the study is that therapists were assessing positively the efficacy of treatments they deliver themselves. Future research can use colleagues' assessments of the client' progress and treatment outcomes. Moreover, setting up more comprehensive treatment efficacy criteria would enable an additional outlook. For instance, treatment success indices may include participation in treatment sessions, completion of treatment' homework (e.g. in-vivo exposure, practice of breathing techniques), implementation of therapist' recommendations, daily use of tools learned during treatment, and several client' self-reports regarding: reduction in symptoms, confidence in daily coping, enhanced recovery ability from stress and indications that significant others are aware of behavior change. Future studies should also examine gender in the context of stress intervention in ongoing threat and study whether some techniques may be effective as function of gender.

This is the first attempt to look into what clinicians suggest as best practices under the conditions of ongoing exposure to military conflict. It is clear that the described process is only one step toward a design of a large scale research aiming to recommend to clinicians working in under such conditions, EBT methods.

\section{Acknowledgement}

We would like to thank all professional staff from the Sha'ar HaNegev Psychological Services Center, Israel, for their participation in the focus group and for their insights.

\section{References}

Al-Krenawi, A., Graham, J. R., \& Kanat-Maymon, Y. (2009). Analysis of trauma exposure, symptomatology and functioning in Jewish Israeli and Palestinian adolescents. The British Journal of Psychiatry, 195, 427. http://dx.doi.org/10.1192/bjp.bp.108.050393

Ayalon, O., \& Lahad, M. (2000). Life on the Edge: Coping with Stress of War and Peace. Haifa: Nord Publications.

Baum, N. (2013). Professionals' Double Exposure in the Shared Traumatic Reality of Wartime: Contributions to Professional Growth and Stress. British Journal of Social Work. http://dx.doi.org/10.1093/bjsw/bct085

Bisson, J. I., Roberts, N. P., Andrew, M., Cooper, R., \& Lewis, C. (2013). Psychological therapies for chronic post-traumatic stress disorder (PTSD) in adults. The Cochrane Database Systematic Review. http://dx.doi.org/10.1002/14651858.CD003388.pub4 


\section{MInstitute ${ }^{\text {Macrothink }}$}

International Journal of Social Work ISSN 2332-7278

Becker, C. B., Zayfert, C., \& Anderson, E. (2004). A survey of psychologists' attitudes towards and utilization of exposure therapy for PTSD. Behaviour Research and Therapy, 42, 277-292. http://dx.doi.org/10.1016/S0005-7967(03)00138-4

Barker, C., Pistrang, N., \& Elliott, R. (2003). Research methods in clinical psychology: An introduction for students and practitioners. England: John Wiley \& Sons.

Besser, A., \& Neria, Y. (2009). PTSD symptoms, satisfaction with life, and prejudicial attitudes toward the adversary among Israeli civilians exposed to ongoing missile attacks. Journal of Traumatic Stress, 22, 268-275. http://dx.doi.org/10.1002/jts.20420

Breslau, N. (2009). The epidemiology of trauma, PTSD, and other posttrauma disorders. Trauma, Violence, \& Abuse, 10, 198. http://dx.doi.org/10.1177/1524838009334448

Cahill, S. P., Foa, E. B., Hembree, E. A., Marshall, R. D., \& Nacash, N. (2006). Dissemination of exposure therapy in the treatment of posttraumatic stress disorder. Journal of Traumatic Stress, 19, 597-610. http://dx.doi.org/10.1002/jts.20173

Cloitre, M. (2009). Effective psychotherapies for posttraumatic stress disorder: a review and critique. CNS Spectr, 14, 32-43.

Cohen, E., Pat-Horenczyk, R., \& Haar-Shamir, D. (2013). Making room for play: An empirically-informed intervention for toddlers and families under rocket fire. Journal of Clinical Social Work. http://dx.doi.org/10.1007/s10615-013-0439-0

Cohen, J. A., Mannarino, A. P., \& Knudsen, K. (2004) Treating childhood traumatic grief: A pilot study. Journal of the American Academy of Child \& Adolescent Psychiatry, 43, 1225-1233. http://dx.doi.org/10.1097/01.chi.0000135620.15522.38

Cukor, J., Olden, M., Lee, F., \& Difede, J. A. (2010). Evidence based treatments for PTSD, new directions, and special challenges. Annals of the New York Academy of Sciences, 1208, 82-89. http://dx.doi.org/10.1111/j.1749-6632.2010.05793.x

Cukor, J., Spitalnick, J., Difede, J. A., Rizzo, A., \& Rothbaum, B. O. (2009). Emerging treatments for PTSD. Clinical Psychology Review, 29, 715-726. http://dx.doi.org/10.1111/j.1749-6632.2010.05793.x

Daniel, T., \& Mcleod, J. (2006). Weighing up the evidence: A qualitative analysis of how person-centred counsellors evaluate the effectiveness of their practice. Counselling and Psychotherapy Research, 6(4), 244-249. http://dx.doi.org/10.1080/14733140601024762

Department of Veterans Affairs/Department of Defense. (2004). VA/DoD clinical practice guideline for the management of posttraumatic stress, version 1.0. Washington, DC: Veterans Health Administration, Department of Defense. [Online]:
[ http://www.healthquality.va.gov/ptsd/ptsd_full.pdf (April 23, 2014)

Diamond, G. M., Lipsitz, J. D., Fajerman, Z., \& Rozenblat, O. (2010). Ongoing traumatic stress response (OTSR) in Sderot, Israel. Professional Psychology: Research and Practice, 41, 19-25. http://dx.doi.org/10.1037/a0017098 


\section{Macrothink}

International Journal of Social Work ISSN 2332-7278 2014, Vol. 1, No. 1

Farchi, M., \& Gidron, Y. (2011). [Posttraumatic symptoms among children from Sderot]. Unpublished raw data.

Farhi, E., Lauden, A., Ifargan, G., \& Fariger, M. D. (2008). Impact of Continuous Terrorism Missile Aattacks on the Emotional and Physical Strength of the Local Population in Sderot, Israel. Epidemiology, 19, S106.

Foa, E. B., Keane, T. M., Friedman, M. J., \& Cohen, J. A. (2009). Effective treatments for posttraumatic stress disorder: Practice guidelines from the International Society for Traumatic Stress Studies. Second Edition. New York: Guilford Publications.

Foa, E. B., Gillihan, S. J., \& Bryant, R. A. (2013). Challenges and Successes in Dissemination of Evidence-Based Treatments for Posttraumatic Stress Lessons Learned From Prolonged Exposure Therapy for PTSD. Psychological Science in the Public Interest, 14(2), 65-111. http://dx.doi.org/10.1177/1529100612468841

Freedman, S., \& Tuval-Mashiach, R. (2006). Mental Health Issues and Implications of Living under Ongoing Terrorist Threats. In L. A. Schein, H. I. Spitz, G. M. Burlingame, P. R. Muskin, (Eds), Psychological Effects of Catastrophic Disasters: Group Approaches to Treatment (p. 33-59). Howarth Press. http://dx.doi.org/10.1177/1529100612468841

Frueh, B. C., Grubaugh, A. L., Cusack, K. J., \& Elhai, J. D. (2009). Disseminating evidence-based practices for adults with PTSD and severe mental illness in public-sector mental health agencies. Behavior Modification, 33, http://dx.doi.org/10.1177/0145445508322619

Gray, M. J., Elhai, J. D., \& Schmidt, L. O. (2007). Trauma Professionals' Attitudes Toward and Utilization of Evidence-Based Practices. Behavior modification, 31, 732. http://dx.doi.org/10.1177/0145445507302877

Hill, C. E., \& Lambert, M. J. (2004). Methodological issues in studying psychotherapy processes and outcomes. Bergin and Garfield's handbook of psychotherapy and behavior change, $5, \quad 84-135$ [Online] Available: https://moodle.umons.ac.be/pluginfile.php/53661/mod_resource/content/1/handbook_of_psyc hotherapy_ch_4.pdf(April 9, 2014)

IDF Blog. (2014). Rocket Attacks on Israel from Gaza. [Online] Available: http://www.idfblog.com/facts-figures/rocket-attacks-toward-israel/ (March 3, 2014)

Israel Trauma Coalition (ITC). (2012). Working in an ongoing traumatic situation, what helps clinicians and what works for clients? Jerusalem; The Israel Trauma Coalition Oct. 2012 (unpublished)

Lahad, M., \& Leykin, D. (2010). Ongoing exposure versus intense periodic exposure to military conflict and terror attacks in Israel. Journal of Traumatic Stress, 6, 691-698. http://dx.doi.org/10.1002/jts.20583

Lahad, M. (2000). Darkness over the abyss: supervising crisis intervention teams following disaster. Traumatology, 6, 273. http://dx.doi.org/10.1177/153476560000600403 


\section{Macrothink}

International Journal of Social Work ISSN 2332-7278 2014, Vol. 1, No. 1

Lahad, M., Doron., M., Farchi., M., \& Leykin, D. (2011). Treating PTSD among children using SEE FAR CBT. Unpublished manuscript.

Lahad, M., Farhi, M., Leykin, D., \& Kaplansky, N. (2010). Preliminary study of a new integrative approach in treating post traumatic stress Disorder: SEE FAR CBT. The Arts in Psychotherapy, 37, 391-399. http://dx.doi.org/10.1016/j.aip.2010.07.003

Mahoney, M. J. (1977). Cognitive therapy and research: A question of questions. Cognitive therapy and Research, 1(1), 5-16. http://dx.doi.org/10.1007/BF01173501

Mendes, D. D., Mello, M. F., Ventura, P., de Medeiros Passarela, C., \& de Jesus Mari, J. (2008). A systematic review on the effectiveness of cognitive behavioral therapy for posttraumatic stress disorder. The International Journal of Psychiatry in Medicine, 38, 241-259. http://dx.doi.org/10.2190/PM.38.3.b

National Institute for Clinical Excellence (NICE). (2005). The management of PTSD in adults and children in primary and secondary care. Wilshire, United Kingdom: Cromwell Press. $\begin{array}{llll}\text { Retrieved } & \text { April } & 23^{\text {rd }} & 2014\end{array}$ http://www.nice.org.uk/nicemedia/live/10966/29772/29772.pdf

Pat-Horenczyk, R., Schiff, M., \& Doppelt, O. (2006). Maintaining Routine despite Ongoing Exposure to Terrorism: A Healthy Strategy for Adolescents? Journal of Adolescent Health, 39, 199-205. http://dx.doi.org/10.1016/j.jadohealth.2005.11.021

Seidler, G. H., \& Wagner, F. E. (2006). Comparing the efficacy of EMDR and trauma-focused cognitive-behavioral therapy in the treatment of PTSD: a meta-analytic study. Psychological Medicine, 36, 1515-1522. http://dx.doi.org/10.1017/S0033291706007963

Shalev, A., Tuval, R., Frenkiel-Fishman, S., Hadar, H., \& Eth, S. (2006). Psychological responses to continuous terror: A study of two communities in Israel. American Journal of Psychiatry, 163(4), 667-673. http://dx.doi.org/10.1176/appi.ajp.163.4.667

Tanielian T., \& Jaycox L. H. (Eds.) (2008). Invisible Wounds of War, Psychological and Cognitive Injuries, Their Consequences, and Services to Assist Recovery. Santa Monica Cal.
RAN
$\mathrm{D}$
Corp.
[Online]
Available:

http://www.rand.org/content/dam/rand/pubs/monographs/2008/RAND_MG720.pdf (April 9, 2014)

Thabet, A. A., Ibraheem, A. N., Shivram, R., Winter, E. A., \& Vostanis, P. (2009). Parenting support and PTSD in children of a war zone. International Journal of Social Psychiatry, 55, 226. http://dx.doi.org/10.1177/0020764008096100

Thabet, A. A., Tawahina, A. A., El Sarraj, E., \& Vostanis, P. (2008). Exposure to war trauma and PTSD among parents and children in the Gaza strip. European Child \& Adolescent Psychiatry, 17, 191-199. http://dx.doi.org/10.1007/s00787-007-0653-9

van Minnen, A., Hendriks, L., \& Olff, M. (2010). When do trauma experts choose exposure therapy for PTSD patients? A controlled study of therapist and patient factors. Behaviour Research and Therapy, 48, 312-320. http://dx.doi.org/10.1016/j.brat.2009.12.003 


\section{Macrothink}

International Journal of Social Work

ISSN 2332-7278 2014, Vol. 1, No. 1

Waller, G. (2009). Evidence-based treatment and therapist drift. Behaviour Research and Therapy, 47, 119-127. http://dx.doi.org/10.1016/j.brat.2008.10.018

White, B. (2013). Quarterly Update on Conflict and Diplomacy: 16 February-15 May 2013. Journal of Palestine Studies, 42(4), 126-149. http://dx.doi.org/10.1525/jps.2013.42.2.128

\section{Copyright Disclaimer}

Copyright reserved by the author(s).

This article is an open-access article distributed under the terms and conditions of the Creative Commons Attribution license (http://creativecommons.org/licenses/by/3.0/). 